\title{
VIBRATION ANALYSIS BY THE GABOR TRANSFORM METHOD
}

This paper deals with description and application of the Gabor transform for vibration analysis. This transform belongs to the group of linear time-frequency processes. Thanks to its properties, it may be successfully used in the area of non-stationary and transitional signals describing various natural processes. Use for analysis of vibrations caused by railway transport represents quite an interesting application area of this transform. This paper contains mathematical analysis of the transform, a case study and practical experience obtained, as well as recommendations for its practical use.

Keywords: railway switches, rail fastenings, vibration, time-frequency analysis, linear time-frequency transform

\section{Introduction}

Generally, the time series (measured signals) analysis may be implemented in the time domain, in the frequency, or the timefrequency area. Selection of the appropriate domain depends on the signal type, or the analysis type, and the required result.

The information on any technical, physical or other processes is represented by the time changes in the immediate value of the appropriate physical quantity described by the signal [1]. At the time level, there are quite a lot of methods that may be applied to the measured signal. Among the best-known, there are various sorting methods, searching for local and global minimum and maximum values relative to time, correlation analysis, analysis of signal attenuation and, in particular, various statistical methods. However, direct interpretation of time amplitude representation is neither easy nor expedient in many applications. That is why the signal transformation from the time into the frequency domain is often used. The Fourier transform is the most frequently employed method of conversion from the time to the frequency domain. If the measured signal is a non-stationary one (the frequency content changes in time), the usual process of frequency analysis may not be used for interpretation (localization of frequency components), but other transformation processes and calculation methods should be employed.

\section{Time-frequency analysis}

The so-called time-frequency transform is determined for connection of information that may be obtained from the time and frequency domains. They enable determination of the frequency behaviour in time or time distribution of the frequency spectrum. It means that one of possible methods of data processing is use of the so-called time-frequency transforms. Linear time-frequency transforms represent a very important group of the time-frequency processes. Decomposition of the analysed signal into elementary time-frequency units is a basic principle of calculation of all the linear time-frequency processes. It means that the signal may be presented in the time-frequency domain as $N$-multiple sum in accordance with the following equation $[2,3]$

$x(t)=\sum_{k=0}^{N-1} a_{k} \cdot \phi_{k}(t)$

where $x(t)$ is the signal, $\phi_{k}(t)$ are time-frequency units (basic functions) and $a_{k}$ are the corresponding coefficients. The timefrequency position of the basic functions and the amplitude of their coefficients describe the signal in the time-frequency domain. A certain variant of the Short-time Fourier Transform, i.e. the Gabor transform, represents one of interesting processes from the group of linear time-frequency processes.

The Short-time Fourier Transform (STFT) localizes the frequency components in time with a constant (linear) discrimination. The basic idea of this method consists in portioning the signal into sufficiently small samples that may

\footnotetext{
* ' $\mathrm{J}$ aroslav Smutny, ${ }^{1}$ Daniela Vukusicova, ${ }^{1}$ Viktor Nohal, ${ }^{2}$ Herbert Seelmann

${ }^{1}$ Institute of Railway Structures and Constructions, Faculty of Civil Engineering, Brno University of Technology, Brno

${ }^{2}$ Rail Data Services Austria GmbH \& Co KG, Wien, Austria

E-mail: smutny.j@fce.vutbr.cz
} 
be thought of as being stationary (or ergodic). This is done by multiplying the signal by a certain window function. The Fourier transform is applied to the resulting cut-out part of the function with the window moving as time goes by. In this way the short time Fourier sort of trades off the time representation for a frequency one. It may be defined by the following integral $[3,4]$

$\operatorname{STFT}(\tau, f)=\int_{-\infty}^{\infty}\left[x(t) \cdot g^{\cdot}(t-\tau)\right] \cdot e^{-j \cdot 2 \cdot \pi \cdot f \cdot t}$

where $g$ is a window function, * denotes the complex conjunction, $t$ is time, $\tau$ the time shift of the window, $x(t)$ the signal function and $\operatorname{STFT}(\tau, \mathrm{f})$ its time-frequency representation. Equation (2) suggests that, generally, the short time Fourier transform is linear and complex. If a discrete data sequence is processed, integral Equation (2) has to be modified into a summation form according to Equation (3) as a function of frequency $f=k . \Delta f$ and shift $\tau=r . \Delta t$

$\operatorname{STFT}(r, f)=\sum_{n=0}^{N-1} x(n) \cdot g[n-r] \cdot e^{\frac{-j \cdot 2 \cdot \pi \cdot k \cdot n}{M}}$

where $r=0,1,2, \ldots, \frac{N-M}{S}-1$ and $k=0,1,2, \ldots, M$. Here, $N, M$, and $S$ denote the lengths of the data sequence, window sequence and shift, respectively. It should also be noted that the window segments may overlap.

The time-frequency plotting of the short-time Fourier transform is subject to certain limitations. The resulting time and frequency resolutions are limited by the Heisenberg's uncertainty principle [4].

$$
\Delta t \cdot \Delta \omega=\text { const }
$$

The time and frequency resolution cannot be infinitely small at the same time and their proportion can be expressed by the above principle of indeterminacy. The signal component cannot be represented by a point in a time-frequency space. In a given time-frequency domain, its position inside the rectangle $\Delta t . \Delta \omega$ can be determined. The relationship between the time period $\Delta t$ of the window function $g(t)$ and the bandwidth $\Delta \omega$ of the window function $G(\omega)$ in the frequency plane is given by the following equations. For $\Delta t$, we have:

$$
\Delta t^{2}=\frac{\int_{-\infty}^{\infty}\left(t-t_{0}\right)^{2} \cdot|g(t)|^{2} \cdot d t}{\int_{-\infty}^{\infty}|g(t)|^{2} \cdot d t}
$$

where $t_{0}$ can be calculated using the equation

$$
t_{0}=\frac{\int_{-\infty}^{\infty} t \cdot g(t)^{2} \cdot d t}{\int_{-\infty}^{\infty}|g(t)|^{2} \cdot d t}
$$

Note that the denominator in Equation (5) expresses the energy of the weighing window function $g(t)$. Equations (5) and (6) are analogous to those of the probability theory defining mean value and variance. By analogy, the bandwidth in the frequency domain can also be calculated using the equation

$\Delta \omega^{2}=\frac{\int_{-\infty}^{\infty}\left(\omega-\omega_{0}\right)^{2} \cdot|G(\omega)|^{2} \cdot d \omega}{\int_{-\infty}^{\infty}|G(\omega)|^{2} \cdot d \omega}$

where $\omega_{0}$ is given as

$\omega_{0}=\frac{\int_{-\infty}^{\infty} \omega \cdot|G(\omega)|^{2} \cdot d \omega}{\int_{-\infty}^{\infty}|G(\omega)|^{2} \cdot d \omega}$

Thus, the result of the Short-time Fourier transform is given by the choice of the window function and its width.

The Gabor transform is a special-type of the short-time Fourier transform. It uses a Gauss function to partition the signal into sufficiently small samples, which can be thought of as stationary (or ergodic). The use of a Gauss function brings some advantages. Particularly useful is that it generates the least timefrequency window possible given the Heisenberg's uncertainty principle. The Gauss function and its Fourier transform are defined as follows [4]:

$g(t)=\frac{1}{S} \cdot e^{\frac{-\pi \cdot t^{2}}{s^{2}}}$

$G(\omega)=\frac{1}{\sqrt{\pi}} \cdot e^{\frac{-s^{2} \cdot \omega^{2}}{4 \cdot \pi}}$

where $g(t)$ is the Gauss function, $G(\omega)$ its Fourier transform, $s$ is the width of the Gauss function (scale) and $\omega$ is frequency. Substituting into (5) and (7) yields the following equations for the parameters $\Delta t$ and $\Delta \omega$ of the window function:

$$
\begin{aligned}
& \Delta t^{2}=\frac{s^{2}}{4 \cdot \pi} \\
& \Delta \omega^{2}=\frac{\pi}{s^{2}}
\end{aligned}
$$

Thus, using (11) and (12), we can define the Heisenberg's uncertainty principle for the Gabor transform by:

$$
\Delta t \cdot \Delta \omega=\frac{1}{2}
$$

The Gabor transform is among the basic and fast methods used the for time - frequency analysis of signals. Its precision and fitness are largely dependent on the width of the Gauss function used and on the way the neighbouring segments overlap.

Plotting the values of the Gabor transform into a graph will provide spectrograms. These may be displayed in a $3 \mathrm{D}$ space 


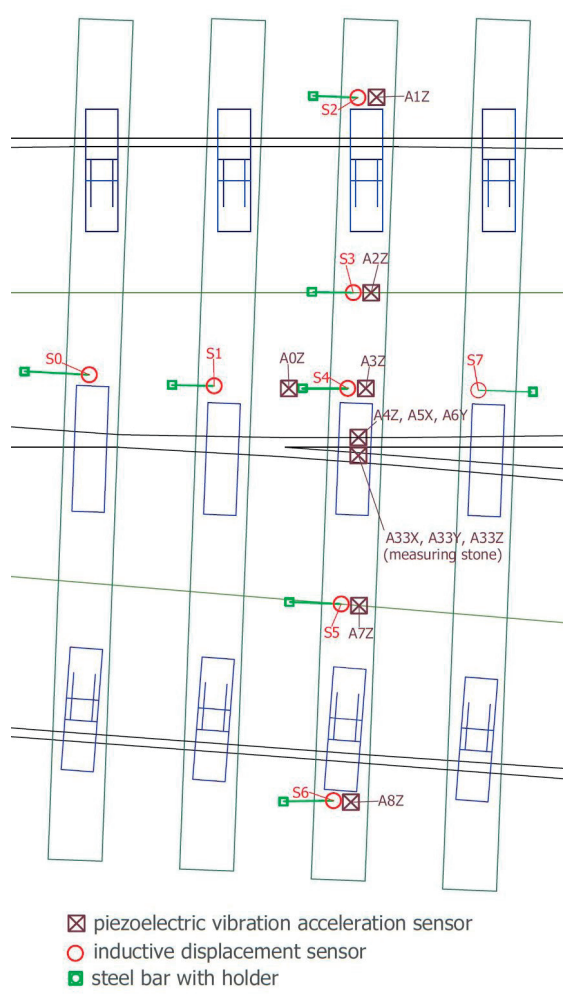

Figure 1 Sensor placement methodology

(frequency, time, amplitude or frequency, time, spectral density). The axonometric projection is very clear but, in many cases, may lead to ambiguities. This is the reason why the two-dimensional mapping is used by means of density spectrograms in which a colour shade is assigned to each amplitude or spectral function value. Using iso-lines is another option. In some sophisticated cases, it may be of some use to complete the time-frequency plot of a spectrum by frequency and time sections. In this way, a strong graphical support of the time-frequency analysis is obtained.

\section{Case study}

Hereafter we present use of the Gabor transform in the area of railway engineering. It is a transient process analysis, vibration analysis.

Permanent pressure to increase the transport speed and the operational load on the railway resulted in huge development of new technologies. The decision on modernization of the corridors became the impulse for development of all areas of the railway transport, either in the area of vehicles or infrastructure. In accordance with the above-mentioned trend, application of new experimental processes should continue for evaluation of the quality and suitability of individual constructional solutions.

The superstructure vibrations are influenced by its quality, operational and technical conditions, climatic events and, most

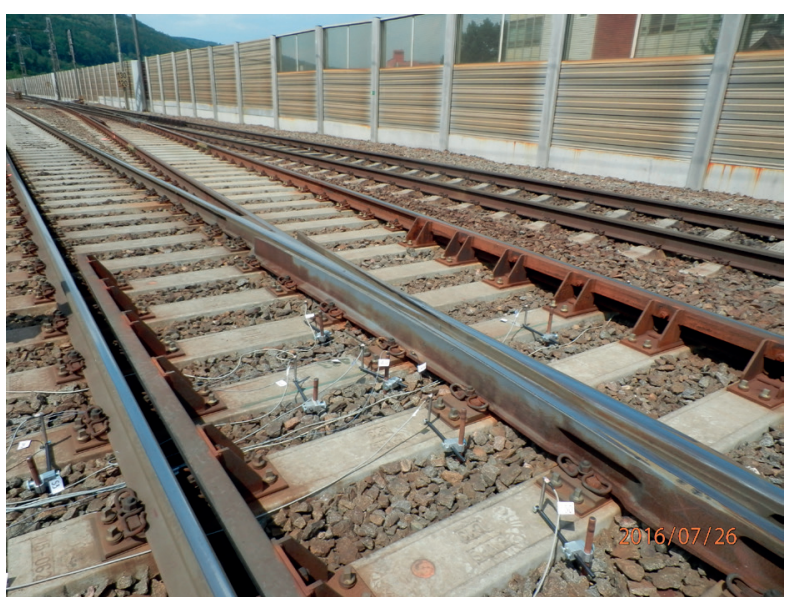

Figure 2 View of the measurement site

importantly, by dynamic load on the railway vehicle axle. The fact also relates to construction of the railway switches representing the key points of the railway roads.

The measurement methodology, certified by the Ministry of Transport of the Czech Republic is available for measurement of dynamic effects influencing the railway switches. The certified methodology was developed by the team of engineers of Brno University of Technology, Institute of Railway Structures and Constructions, under the leadership of prof. Smutny [5]. The methodology (see Figure 1) consists of three parts [6].

The first of them deals with measurement of the construction movements. Particularly, it is an analysis of shifts of individual parts of the switches under load. The second part deals with distribution of vibrations around the crossing nose and replacement of the switches and namely with effect of the vibrations on the bed of ballast. Within the third part, measurement of the force application is performed. Application of the time-frequency analysis of the obtained data forms a part of the interpretation methodology.

The two switches, namely switches No. 3 and No. 4, were selected for presentation of the Gabor transform application in the analysis process of dynamic forces from the train units. The selected switches are located on the "trebovsky" throat of the Usti nad Orlici station and it represents a simple crossover (Figure 2). Except for the elasticity of the fastening system [7], the two switches are identical. The No. 3 switch is fitted with a new rail fastening type that is more elastic and better absorbs vibrations transmitted from the rail to the bed of ballast.

Passage of the unit SC Pendolino at $160 \mathrm{~km} \cdot \mathrm{h}^{-1}$ was chosen for comparison of dynamic stress in both of the switches. Due to shortness of the paper the authors concentrate on analysis of the data read from the sensor located on the sleeper under the crossing nose. It provides the vertical components of acceleration of vibrations in the fixation point. The analysis is given in figures consisting of three diagrams. The upper diagram describes behaviour of acceleration of the vibrations in time. The diagram 
Pendolino set, $160 \mathrm{~km} / \mathrm{h}$, switch no. 3
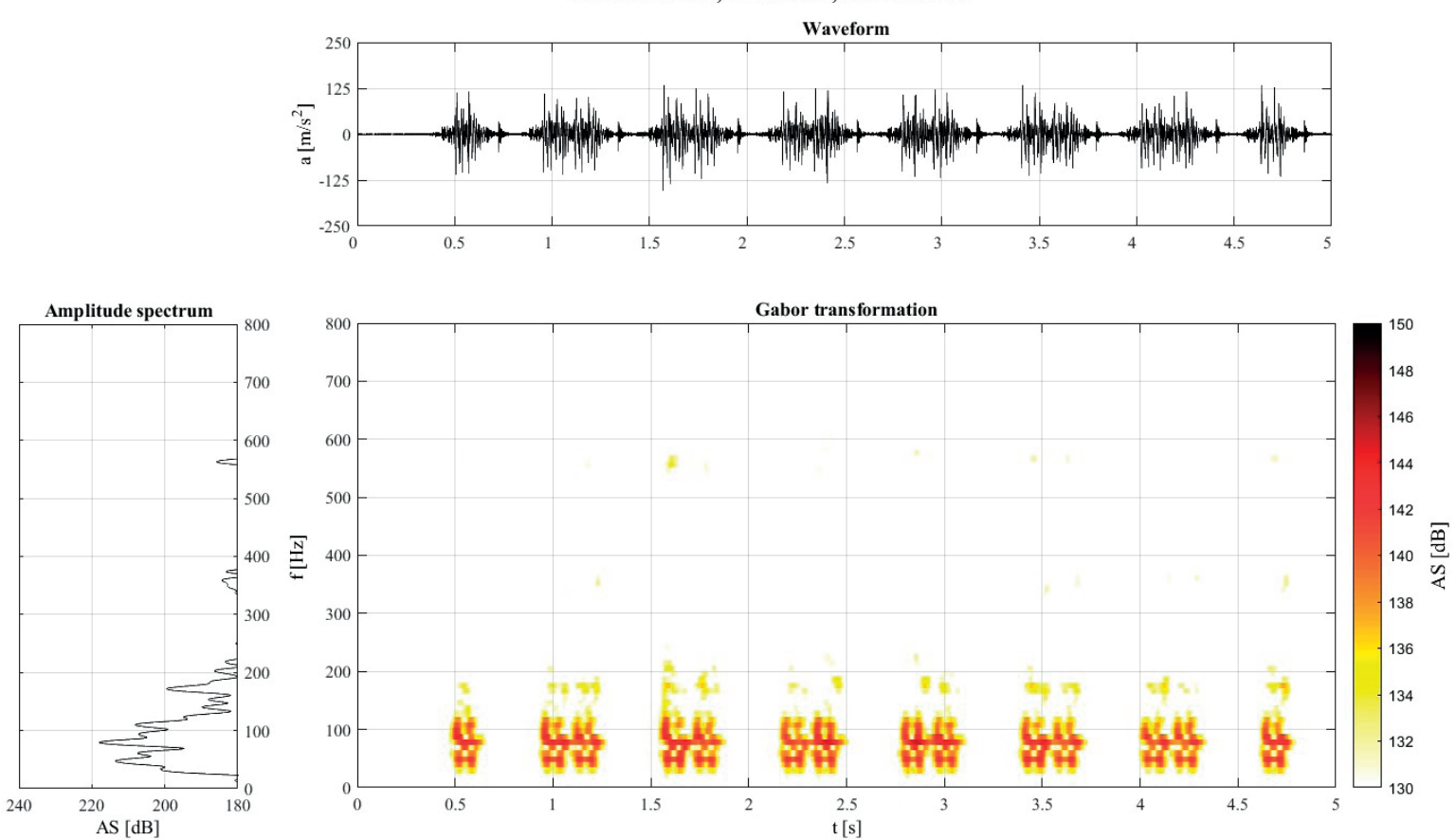

Figure 3 Time history of acceleration (up), amplitude spectrum (left) and jointed time - frequency spectrum in the vertical direction (right) for switch No. 3

to the left gives the amplitude spectrum of the vibration response calculated using the Fourier transform. The diagram in the middle gives the 3D image of the time-frequency behaviour of the vibration response amplitude spectrum. The values of the vibration acceleration in the decibel scale are shown in the middle graph as different colour areas, while the maximum value is black.

It is obvious from the diagrams of acceleration behaviour in time (Figures 3 and 4, the upper diagram) that the higher acceleration values were obtained in the No. 4 switch (usual rail fastening type) if compared to the No. 3 switch with the new rail fastening type. In this case the diagrams show the worse geometry of the transfer from the wing rail to the crossing nose, the higher dynamic shock and this way the worse absorption of the vibration source, i.e. passage of the wheel through the crossing nose.

The diagrams on the left (Figures 3 and 4 ) show the amplitude spectra of the vibration acceleration, calculated from the time signals using the Fourier transform. The amplitude spectra are presented in the frequency interval from $1 \mathrm{~Hz}$ to $800 \mathrm{~Hz}$, while the frequency values up to approx. $600 \mathrm{~Hz}$ are important for dynamic stress on the permanent way construction parts.

From the amplitude spectrum diagram of the No. 3 switch it is obvious that there are four important frequency bunches in the spectra. The first one is from approx. $30 \mathrm{~Hz}$ to $60 \mathrm{~Hz}$ with maximum at $40 \mathrm{~Hz}$; the second one is in the interval from $75 \mathrm{~Hz}$ to $100 \mathrm{~Hz}$ with maximum at $80 \mathrm{~Hz}$; the third one from $100 \mathrm{~Hz}$ to $130 \mathrm{~Hz}$ with maximum at $110 \mathrm{~Hz}$, and the fourth one from approx. $180 \mathrm{~Hz}$ to $210 \mathrm{~Hz}$ with maximum at $180 \mathrm{~Hz}$. It is obvious from the frequency spectrum, obtained from the signal measured at the No. 4 switch, that the frequency bunches are wider, with higher maximum spectrum values. It may be concluded from the spectra that they show more complex vibration processes in the area of passage of the wheels through the crossing nose and higher energy to the disadvantage of the switch with the usual rail fastening system (No. 4).

The colour spectrograms calculated by application of the Gabor transform were used for evaluation of vibrations in the time-frequency domain. It concerns the diagrams in the middle of the figures No. 3 and No. 4. Extreme values of the vibration amplitude spectrum are depicted in the diagram as small red areas corresponding to time intervals of the wheel (axle) passage over the sensors. It is obvious from the diagrams that the time occurrence of important frequency components in the spectrum is different in the two constructions. In the diagrams, it may be identified at which moment the appropriate process occurred (which wheel, axle participates) and which frequency components relate to the process. It means that the linear time-frequency analysis provides the true and correct data complementing the conclusions obtained from the time and frequency analysis.

Finally, we may state that from both, application of the whole methodology and the results obtained from the sensor located on the sleeper under the crossing nose, the positive effect of the more elastic rail fastening is evident. It better absorbs the peak values of the vibrations acceleration upon transfer from the wing rail to the sleeper and the bed of the ballast. 


\section{kanNllkacle}
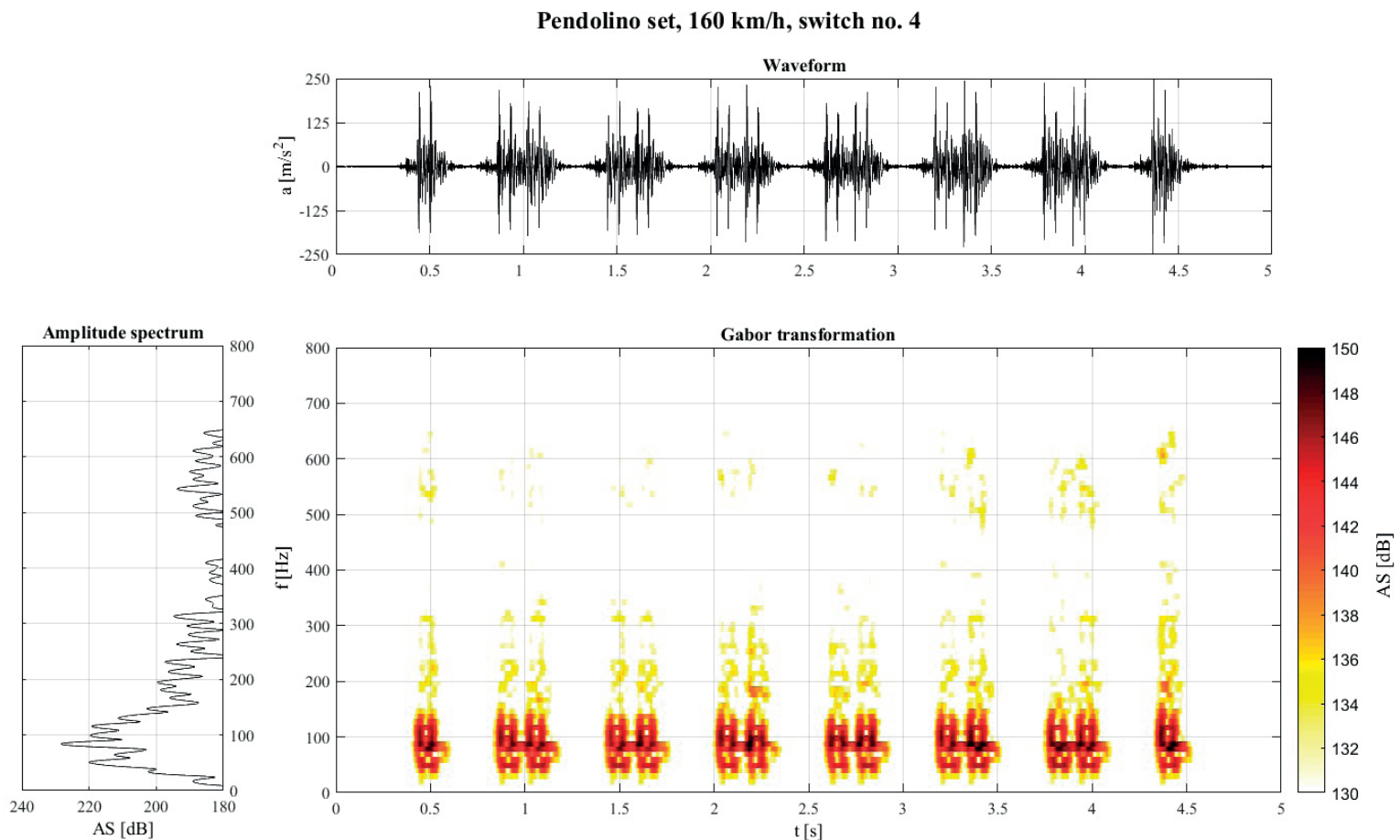

Figure 4 Time history of acceleration (up), amplitude spectrum (left) and jointed time - frequency spectrum in the vertical direction (right) for switch No. 4

\section{Conclusions}

If compared to other methods, the time-frequency analysis enables time localization of the frequency components contained in the measured signals. This way it enables overall view of the transition or non-stationary characteristics of the analysed processes.

As a benefit, the Gabor transform enables rapid calculation and satisfactory time-frequency resolution. A certain disadvantage of the method is the fact that the resulting resolution in time and frequency is limited by the so-called Heisenberg uncertainty principle. It means that the signal frequency component may not be presented as a point in the time-frequency space, but its position may be determined only inside the rectangle $\Delta t$. $\Delta f$ in the given time-frequency domain. It should be noted that $\Delta t$ represents a minimum time interval or a time step, while $\Delta f$ represents the minimum frequency interval or the frequency step. In spite of the small disadvantage the Gabor transform may be recommended as one of the basic methods of the analysis of non-stationary signals. It must be said that in practice the process often proves successful if the signal is processed with the Gabor transform with additional frequency section first. This enables to obtain the basic information about the given signal in the time, frequency and time-frequency domains. An analysis of the obtained results may serve as a basis for selection of a more advantageous transformation for subsequent analysis.

Finally, it may be stated that the used methodology provides good results and conclusions. The measured and calculated values are accurate enough and provide sufficient informative value. It may also be stated that the up-to-date signal analysis means that the Gabor transform, made the first-rate processing of the measurements possible. Based on the interpretation of results, it may be said that the new rail fastening system (with different rail pads, which reduce the overall stiffness of the track) in the No. 3 switch, seems to be very promising. It shows a positive effect on decrease of dynamic effects on the sleeper and the bed of ballast.

\section{Acknowledgments}

The research reported in this paper has been supported through the research project FAST-S-17-4576, "Study of dynamic phenomena in railway superstructure construction by selected methods of linear time-frequency analysis", and LO1408, "AdMaS UP, Advanced Materials, Structures and Technologies”, funded by Ministry of Education, Youth and Sports of the Czech Republic. 


\section{References}

[1] MORAVCIK, M.: Analysis of Vehicle Bogie Effects on Track Structure-Nonstationary Analysis of Dynamic Response. Communications - Scientific Letters of the University of Zilina, 13 (3), 33-40, 2011.

[2] SMUTNY, J.: Measurement and Analysis of Dynamic and Acoustic Parameters of Rail Fastening. NDT \& E International Independent Nondestructive Testing and Evaluation, 37 (2), 119-129, 2004.

[3] SMUTNY, J., PAZDERA, L.: New Techniques in Analysis of Dynamic Parameters Rail Fastening. INSIGHT, 46 (10), $612-615$, 2004.

[4] SMUTNY, J., PAZDERA, L.: The Experimental Analysis of Dynamic Processes Related to Railway Transport, monography. Academic publisher CERM, Brno, 2012.

[5] SMUTNY, J., PAZDERA, L., VUKUSIC, I.: Dynamic Effects on Switches Evaluation. Certified Methodology, Ministry of Transportation, 124/2014-710-VV/1, 2014.

[6] SMUTNY, J., SADLEKOVA, D.: The Vibration Analysis by Margenau - Hill Transformation Method, Communications - Scientific Letters of the University of Zilina, 16 (4), 123-127, 2014.

[7] SMUTNY, J.; PAZDERA, L.; TOMAndL, V.; SEElmanN, H.: Assessment of Dynamic Parameters of Rail Fastening. Communications - Scientific Letters of the University of Zilina, 18 (3), 65-70, 2016. 